\title{
Saliva, hair, tears, and other biological materials obtained non-invasively for diagnosis in pregnancy: a literature review
}

\author{
Aleksandra Zygula, Przemyslaw Kosinski, Miroslaw Wielgos \\ 1st Chair and Department of Obstetrics and Gynecology, Medical University of Warsaw, Poland
}

\begin{abstract}
As medical technology evolves, clinicians are increasingly choosing relatively painless non-invasive methods of patient diagnosis and treatment. There are two principles behind this: greater patient comfort and lower cost. Tears, hair, saliva, urine, and faeces can replace blood for diagnosis. The varied constituents in these biological materials can serve as biomarkers for the detection of both local and systemic diseases. In this paper, we review a range of diagnostic techniques - all using biological material obtained via non-invasive procedure - for detecting medical conditions in pregnant women.

PubMed, Medline, Embase, and the Cochrane Library were searched from January 1996 until December 2018. Forty seven studies were included: thirty-five original articles, nine reviews and three meta-analysis.

Analysis showed that saliva, hair, tears, and other biological material — obtained via non-invasive methods — may serve as clinically informative biomarkers. These biomarkers may be used for: toxicology, psychological studies, disease detection, biomonitoring, and drug abuse. The analysis of tears, hair, saliva, urine, and faeces is a safe, noninvasive and useful diagnostic tool within groups of pregnant women, but further investigation is necessary to fully realize the promise of these novel diagnostic tools.
\end{abstract}

Key words: tears; saliva; faeces; urine; hair; noninvasive

Ginekologia Polska 2019; 90, 8: 475-481

\section{INTRODUCTION}

As medical technology evolves, clinicians are increasingly choosing relatively painless non-invasive methods of patient diagnosis and treatment. There are two principles behind this: greater patient comfort and lower cost.

A milestone was achieved recently in fetal medicine: the debut of non-invasive testing via cell-free fetal DNA (cffDNA). Although still considered to be a method for screening rather than diagnosis, in certain cases cffDNA tests may be a useful option, replacing invasive techniques such as amniocentesis or chorionic villous sampling (CVS).

Tears, hair, saliva, urine, and faeces can replace blood for diagnosis. The varied constituents in these biological materials can serve as biomarkers for the detection of both local and systemic diseases. In this paper, we review a range of diagnostic techniques - all using biological material obtained via non-invasive procedure - for detecting medical conditions in pregnant women.

\section{Urine}

Urine is a readily extractable biological fluid. Urine testing is an increasingly common non-invasive means of obtaining clinical data [1]. Such tests have an established part in maternal-fetal medical practice e.g. for detecting and monitoring proteinuria.

Due to its particular physiological function, urine is often characterized by irregular variation between individual persons, or at successive stages for a single individual. Large variations reflect urinary sensitivity to both normal physiological change as well as abnormal pathological change in vivo [1]. In the absence of homeostatic mechanisms - and unlike blood - urinary variation may indicate more change, especially in the early stages of disease. Urine samples can be collected long-term and painlessly. Moreover, urine collection is safer and cheaper than blood collection. Membrane technology [a new technique that absorbs urine proteins found on PVDF (polyvinylidene fluoride membrane), which can then be dried and stored] and other modern techniques, 
have made collecting and storing urine samples even easier. Biomarkers found in urine may indicate the following:

\section{ZIKV infection}

Saliva and urine have been recognized for their value in diagnosing viruses such as Zika [2], which has seen a violent outbreak on a global scale. Zika may present in a milder form — lasting approximately one week - with symptoms such as rash, fever, myalgia, arthralgia, conjunctivitis, headache, and malaise. However, vertical transmission of the Zika virus may present fetal growth restriction, placental insufficiency, microcephaly, CNS, or even fetal death [3]. A recent study described the lower efficiency of experimental transmission for Zika's main assumed vector: Aedes Aegypti, together with signs of probable sexual transmission. This led to consideration of other possible sources for Zika. Bonaldo et al. tested 5 saliva and 9 urine samples of patients from Rio de Janeiro. The results showed infectious Zika virions in the saliva and urine of patients in the acute stage, which may be a key factor in the dissemination of the virus. Bringhal et al. also tested saliva and urine, finding that: $95 \%$ (52/55) of urine samples taken from patients within 5 days of symptom appearance tested positive by RT-PCR; merely 56\% (31/55) of serum samples taken on the same day tested positive by RT-PCR; $82 \%$ (9/11) of urine samples taken > 5 days after symptoms began tested positive by RT-PCR; zero RT-PCR tests for serum samples were positive. Such findings indicate that urine may be the best type of sample to detect the acute stage of Zika. Considering the importance of other nonvectorial transmission routes, the epidemiological relevance of this calls for additional study.

\section{Podocytes: a potential new biomarker for preeclampsia}

There is no straightforward, cheap, dependable, test to predict with high accuracy whether preeclampsia will occur during pregnancy. The priority is to identify biomarkers - which should be non-invasively obtained and easily determined - to improve the efficient detection of this condition.

There is growing evidence that the urinary excretion of viable podocytes may be a useful indicator of preeclampsia. Garovic et al. extracted podocyte cells from urine in preeclamptic women. The study demonstrated a significant relationship between podocyturia and proteinuria. Furthermore, it was shown that the quantity of podocyte cells excreted in urine is a sensitive indicator of renal damage as well as protein excretion among patients with preeclampsia.

Another discovery was that urinary podocyte cells were absent in healthy pregnant women, as well as those presenting hypertension but no other preeclamptic symptoms [4]. Aita et al. [5] noted a strong link between podocyte quantity lost in urine and blood pressure, although not in the case of proteinuria. In 2012, Zhao et al. noticed a reduced expression of podocyte-slit diaphragm proteins - including polarity proteins, podocin, and nephrin - in preeclampsia. Tracing the relationship between urine podocyte cells and preeclampsia may show great promise, nonetheless, additional research is required [6].

\section{SLOS}

Non-invasive urine collection can identify the rarest of genetic fetal diseases. For example, the steroid metabolome, once extracted from maternal urine, can be used to confirm a diagnosis of Smith-Lemli-Opitz Syndrome (SLOS) [7]. The optimum standard for prenatal diagnosis is an increased level of 7-dehydrocholesterol (7-DHC), tested via either gas chromatography or mass spectrometry, and measured from the amniotic fluid after 14 weeks or from chorionic villous samples (CVS) between 10 and 12 weeks. Both amniocentesis and CVS are invasive procedures and risk miscarriage [8]. Consequently, testing specific steroid compounds (dehydroestriol, 7-dehydropregnanetriol, 8-dehydropregnanetriol) in the maternal urine has been suggested as a non-invasive method for diagnosing SLOS [9]. Guo et al. have described how particular concentrations of these steroid compounds present in urine, with each SLOS analyte expressed as a ratio of its natural counterpart, can indicate SLOS between 14 and 22 weeks. This has been confirmed by other research such as Jezela-Stanek et al. [10] who successfully diagnosed prenatal SLOS employing maternal urine steroid ratios.

\section{Faeces}

Inflammatory bowel disease (IBD) is very common among adults of reproductive age [11]. When active, the condition increases risks associated with pregnancy, including preterm birth (PTB) and intrauterine growth restriction [12]. Possessing a recognized clinical tool to monitor IBD during pregnancy is an important, albeit challenging, care factor. In patients who are not pregnant, endoscopy is the optimum technique for assessing IBD severity. According to international guidelines, when an endoscopy is required, a sigmoidoscopy is usually the preferred method, ideally in the second trimester [13]. Employing other methods could be preferential when an endoscopy is unnecessary. Sedatives and extended procedures could harm maternal circulation. Consequently, non-invasive methods are of great value to monitor IBD.

One such method employs faecal calprotectin (FC) [14]. This is an accurate biomarker for mucosal inflammation, which correlates with histological inflammation as well as endoscopic activity [15]. Julsugaard et al. studied 46 pregnant women and found that FC is not affected by physiological changes 
occurring during pregnancy. Furthermore, the combination of the physician's global assessment (PGA) and FC may be the best alternative to endoscopy for measuring disease activity. PGA and FC are also superior to other biomarkers such as the plasma concentration of C-reactive protein (CRP). The study also described how - instead of colonoscopy - FC could be employed to differentiate non-specific IBDs and distinguish them from irritable bowel syndrome [16]. However, no similar study has been carried out during pregnancy and thus additional research is required.

\section{Tears}

Tears are a mixture of proteins, mucins, lipids, salts, and water. A recent proteomic study discovered 1526 proteins in tears [17], meaning that this biological fluid is less complex than either serum or plasma. Because of variations in the lacrimal functional unit (LFU), studying the composition of tears has been suggested as an ideal method for identifying biomarkers linked to different systemic disease ingredients [18]. Recent efforts have sought to identify new tear biomarkers for ocular diseases - e.g. dry eye disease (DED) symptomatic of diabetes - as well as systemic disease. Kan's study was intended to assess tear function in pregnant women with gestational diabetes mellitus (GDM) [19]. The result was surprising: GDM appears to have no negative affect on tear function testing. This could be because of the absence of long-term hyperglycaemia, the presence of which may have affected tear function in pregnant women. As a readily obtainable biological fluid, tears could be employed in future medical testing, although this requires additional study.

\section{Hair}

Different methods of hair analysis are used for: toxicology, anthropological studies, disease detection, biomonitoring, and drug abuse. Human hair is often a useful source of tissue sample as it is readily obtainable as well as stable. Furthermore, sample storage and preparation is straightforward: no specialised preservation method is needed. Hair - depending on its length - may signify a time period of days, months, or even years. Hair analysis is also extensively used in pregnancy.

\section{FASD}

An example of this would be testing maternal hair for ethyl glucuronide. Hair ethyl glucuronide (hEtG) is a biomarker for prenatal alcohol exposure. Alcohol consumption may cause fetal abnormalities such as Fetal Alcohol Spectrum Disorder (FASD) or Intrauterine Growth Restriction (IUGR) [20]. Reducing the number of children harmed by prenatal alcohol exposure (PAE) can be achieved by early, accurate diagnosis of prenatal alcohol consumption, to- gether with targeted harm reduction strategies [21]. One associated challenge concerns the high level of maternal underreporting because of the social stigma around drinking alcohol during pregnancy. Consequently, further screening of biochemical alcohol biomarkers is called for. Because of its high sensitivity and specificity as an indicator of heavy chronic alcohol consumption, hEtG is a promising biomarker. Notwithstanding that, direct ethanol metabolites such as ethyl glucuronide (EtG) serve a key role in confirming $\mathrm{PAE}$, although their utility may be reduced by their shorter half-life in blood and urine. Maternal hair analysis permits retrospective PAE analysis for several months. Screening blood or urine for alcohol biomarkers during the first prenatal visit gives negative results in many pregnant women who cease alcohol consumption upon realizing they are pregnant. Furthermore, hair analysis can indicate dangerous alcohol consumption in the periconceptional period — the critical stage of organogenesis. A meta-analysis of chronic alcohol users by Boscolo-Berto et al. (2014) [22] described an overall sensitivity of $96 \%$ and specificity of $99 \%$ for $\mathrm{hEtG}$. Furthermore, hEtG — as a direct ethanol metabolite - has a higher specificity than other alcohol biomarkers tested in urine or blood. Such biomarkers are known to be affected by various maternal conditions and pregnancy related physiological changes, including liver conditions, hypertension, gestational age, and iron deficiency. In light of these advantages, hEtG would appear to be a reliable biomarker for prenatal alcohol consumption.

\section{Postpartum depression}

Estimates suggest that rates of postpartum depression (PPD) in women range from $10-15 \%$. Studies have long established how PPD can negatively affect the maternal-infant bond, as well as exacerbate outcomes in the child's development - both physically and psychologically [23]. To prevent PPD onset and its negative effects, the early detection of PPD associated factors is necessary. The psychological or behavioural predictors for PPD include: age $<25$, low-earnings, a history of miscarriages or terminations, PMS, antenatal depression, and antenatal anxiety. Although the above predictors are well known, there is a scarcity of biological forecasters. One promising PPD biomarker is hair cortisol. Measuring the levels of this hormone can detect chronic stress occurring in the prior three months. Unlike more typical samples - e.g. blood - situational variables or circadian rhythms do not affect this value [24]. Caparros-Gonzalez et al. studied 44 pregnant women over three pregnancy trimesters and the postpartum period; they employed psychological questionnaires and tested for hair cortisol levels. The study found significant variations in the hair cortisol levels between the groups with and the groups without symptoms of PPD in the first and 
third trimesters [25]. Hair cortisol levels predicted $21.7 \%$ of PPD symptom variation. The research provided evidence that antenatal stress, psychopathological symptoms, and hair cortisol levels at various stages of pregnancy can predict PPD. These results encourage further research aimed at improving maternal care in clinical settings. To summarize, effective prenatal stress screening is both possible and measurable. Consequently, it could be employed on a wider scale to reduce the harm caused by postnatal depression [26].

\section{IVF success rate}

Massey et al. [27] have studied women undergoing IVF treatment, examining the relationship between hair cortisol, salivary cortisol, and pregnancy. Results indicated that there was a significant negative association between concentrations of hair cortisol and clinical pregnancy outcomes $(p=0.017)$. The study offered preliminary evidence that longer-term systemic cortisol could have an effect upon pregnancy outcomes. Consequently, psychological intervention to lower cortisol levels prior to IVF treatment, such as CBT or mindfulness, could improve patient outcomes.

\section{Fetal defects}

Hair sample analysis is more reliable than blood analysis when measuring the concentration and chronic exposure of different metals in individual pregnant women [28]. Hair samples are more stable and easier to collect than blood. Studies on the concentration of metal in hair samples have given valuable insight on the potential origins of congenital defects. For example, a higher titanium level in a woman's hair during periconception was linked with a greater risk of neural tube defects (NTD) in children. This could be partially mitigated by the mother's diet [29]. Furthermore, exposure by the mother to cadmium and arsenic could significantly increase the risk of congenital heart defects (CHDs) in children [30]. An increased aluminum concentration in maternal hair may significantly increase the likelihood of a child being born with a CHD. Such conditions include conotruncal heart defect, septal defect, and right-sided obstructive heart defect [31]. Because of the wide dissemination of the above metals in our $21^{\text {st }}$ century lives, and their potential risk to a developing fetus, women should be strongly encouraged to adjust their diets during periconception and pregnancy. Testing hair samples can indicate when dietary and lifestyle changes should be introduced by pregnant women; the implementation of such changes could lead to a fall in the number of fetal abnormalities.

\section{Predictors of viral suppression in women infected with HIV}

It is recognized that sufficient antiretroviral drugs (ARV) can protect HIV infected pregnant women and decrease the risk of viral transmission to children. Unfortunately, drug compliance can be compromised by psychological changes occurring during pregnancy. This - combined with altered drug barriers - can affect ARV pharmacokinetics, potentially limiting drug exposure. There is therefore an urgent need for an objective means of measuring ARV compliance in pregnant women. The most commonly tested biological sample for repetitive drug use during pregnancy is maternal hair. Hair grows at a rate of $1 \mathrm{~cm}$ per month; it is thereby possible to assess the pattern of drug compliance over three trimesters [32].

PROMOTE was a Ugandan study on HIV-infected pregnant women at 12-28 weeks, who were randomly assigned either lopinavir or efavirenz-based antiretroviral therapy (ART) [33]. The study concluded that measuring ARV concentrations in subjects' hair was a promising method for accurately predicting viral suppression among them. The technique could lower the risk of perinatal HIV transmission during the critical stages of delivery and breastfeeding.

\section{Saliva}

Clinicians first used saliva for medical diagnosis after WW2 [34]. Known as the "mirror of body health", saliva indicates both the local and general health of a patient. It is used to detect key biomarkers of both oral and systemic diseases, as well as indicating an individual's exposure to varied substances. Recently, saliva testing has become a recommended means for detecting body fluid biomarkers, since it is cheap, non-invasive and painless [35]. Furthermore, saliva does not clot, is less prone to potential infection, and can be easily collected, carried, and kept.

\section{Preterm birth}

One tenth of deliveries are preterm; this is the second main cause of neonatal morbidity and mortality. It has proved difficult to identify an optimum biomarker to accurately predict those women who will deliver preterm. Although various current biochemical and biophysical markers have been investigated, a reliably accurate biomarker has yet to be found [36]. Priya et al. [37] tested levels of progesterone in the saliva of asymptomatic high-risk women to predict PTB, comparing this with transvaginal sonographic (TVS) cervix length. The study examined asymptomatic women $(n=90)$ with singleton pregnancies at 24-28 weeks. Subjects had a history of PTB, preterm pre-labour membrane rupture, or late spontaneous miscarriage (at 20-28 weeks). Results showed a positive linear correlation between TVS cervix length and progesterone levels in the saliva. This suggests that the change in TVS cervix length may be indicated by the concentration of progesterone in the saliva. The two tests were compared; salivary progesterone was found to be a better predictor of PTB than cervix length at $<34$ weeks, 
but not at $<37$ weeks. This may suggest that the cervix becomes shorter followed by a decrease in progesterone. It is desirable to isolate the subset of women who could gain maximum benefit from progesterone supplements from a larger set of high-risk asymptomatic women; this can be achieved by measuring progesterone levels in the saliva. The study underlined that a low level of progesterone in the saliva ( $<2575 \mathrm{pg} / \mathrm{mL}$ ) at 24-32 weeks can identify women at risk of PTB.

\section{Monitoring of prenatal smokers}

Smoking is the No.1 cause of avoidable mortality in women and a main preventable cause of negative pregnancy outcomes in high-income economies. The most serious health conditions linked to maternal smoking include: preeclampsia [38], placental abruption, uteroplacental insufficiency, premature membrane rupture, decreased blood flow to the fetus, as well as intrauterine growth retardation [39]. Due to the quantity of severe health conditions linked to smoking, it is essential to both monitor exposure and encourage quitting. Initial smoking assessment in pregnant women is often based on selfreporting via a questionnaire. However, with the increase in stigma around smoking during pregnancy, there is a higher probability that women will not self-report their smoking.

Biochemical verification - by means of non-invasive techniques such as saliva or urine tests - can assess smoking prevalence with greater reliability. Being a matrix, saliva can be destroyed easier than urine; it is therefore used for fast screening of smoking status. Studies performed in high-income economies have shown that pregnant women's self-reporting of smoking can underestimate prevalence by $24-28 \%[40,41]$. The difference may be explained by the raised awareness of the risks of smoking while pregnant, combined with increased stigma towards pregnant smokers.

To conclude, self-reporting is an unreliable measure of smoking in pregnancy. Since it underestimates the number of smokers, additional screening for biomarkers of nicotine intake is required. The concentration of salivary cotinine appears to be an accurate biomarker, which permits active smokers to be separated from passive [42]. An important benefit of cotinine over nicotine as a biomarker for smoking is that approximately $72 \%$ of nicotine is converted into cotinine. The half-life of cotinine averages around $17 \mathrm{~h} \mathrm{com-}$ pared to 2-3 $\mathrm{h}$ for nicotine. The saliva cotinine cut-off level for active smokers was $10 \mathrm{ng} / \mathrm{mL}$ (sensitivity $96 \%$, specificity $95 \%$ ) while for passive smokers it was $1.5 \mathrm{ng} / \mathrm{mL}$ (sensitivity $63 \%$, specificity $71 \%$ ) [43].

\section{GDM and oxidative stress}

Oxidative stress (OS) is thought to be the key factor in the development and progress of gestational diabetes mellitus (GDM). It also plays a role in complications related to GDM, including pregnancy-induced hypertension and intrauterine growth retardation [44]. A useful biomarker for testing oxidative stress levels in GDM populations is saliva [45]. Zygula et al. studied 89 pregnant women $(\mathrm{n}=89 ; \mathrm{GDM}=59$; control = 30). The saliva of each subject was analyzed for malondialdehyde (MDA), total antioxidant capacity (ORAC), and inactivation of aldehyde dehydrogenase (IALDH). Oxidative stress was not found in GDMG1 but was found to be moderate in GDMG2. In GDMG2, higher concentrations of salivary MDA and IALDH were found. Salivary OS was correlated with — and found to be most significant for - ORAC. Salivary ORAC could be a systemic biomarker for oxidative stress [45]. Salivary MDA and the inactivation of ALDH could be biomarkers for OS in the oral cavity. Screening the saliva of pregnant women for OS biomarkers could prove beneficial for oral health problems, gestational diabetes, or even premature delivery [46]. One study found that MT2A present in saliva could be a potential biomarker for the risk of premature delivery. Notwithstanding this, additional research is necessary to confirm these results [46].

\section{Glucose nano-biosensor}

The finger-prick test is part of standard diabetes management, which is painful and inconvenient for selfmonitoring patients. Non-invasive glucose testing could improve compliance and glucose control, as well as decreasing both the complexity and costs of disease management. A new disposable on-chip salivary nano-biosensor has been suggested as a non-invasive technique. The working electrode is functionalized with single-walled carbon nanotubes, chitosan multilayers, gold nanoparticles, and glucose oxidase, with layer-by-layer assembly. The biosensor can detect glucose to $0.1 \mathrm{mg} / \mathrm{dL}$ and gives results in 30 seconds. Studies have shown that these salivary biosensors are a reliable, reproducible and efficient technology, which enables continuous salivary glucose monitoring [47].

\section{SUMMARY}

Saliva, hair, tears, and other biological material - obtained via non-invasive methods - can serve as clinically informative biomarkers. Multiple studies have described how these biomarkers can be used in new techniques for prognosis, laboratory or clinical diagnosis, observation, and management of patients with varied medical conditions. Biological samples - which contain soluble biological markers - have the advantage of being easily collected and stored. These biomarkers can also be used within groups of pregnant women to detect various diseases or medical conditions. However, further research is necessary to fully realise the benefits of these novel approach to diagnostic. 


\section{Conflict of interests}

The authors declare no conflict of interests.

\section{REFERENCES}

1. Jing J, Gao Y. Urine biomarkers in the early stages of diseases: current status and perspective. Discov Med. 2018; 25(136): 57-65, indexed in Pubmed: 29579412.

2. Bonaldo MC, Ribeiro IP, Lima NS, et al. Isolation of Infective Zika Virus from Urine and Saliva of Patients in Brazil. PLoS Negl Trop Dis. 2016; 10(6): e0004816, doi: 10.1371/journal.pntd.0004816, indexed in Pubmed: 27341420.

3. Pomar L, Vouga M, Lambert V, et al. Maternal-fetal transmission and adverse perinatal outcomes in pregnant women infected with Zika virus: prospective cohort study in French Guiana. BMJ. 2018; 363: k4431, doi: 10.1136/bmj.k4431, indexed in Pubmed: 30381296.

4. Garovic VD. The role of the podocyte in preeclampsia. Clin J Am Soc Nephrol. 2014; 9(8): 1337-1340, doi: 10.2215/CJN.05940614, indexed in Pubmed: 25035271.

5. Aita $\mathrm{K}$, Etoh $\mathrm{M}, \mathrm{Hamada} \mathrm{H}$, et al. Acute and transient podocyte loss and proteinuria in preeclampsia. Nephron Clin Pract. 2009; 112(2): c65-c70, doi: 10.1159/000213083, indexed in Pubmed: 19390204.

6. Zhao S, Gu Y, Groome L, et al. OS062. Oxidative stress mediates podocyte injury in preeclampsia. Pregnancy Hypertension: An International Journal of Women's Cardiovascular Health. 2012; 2(3): 210-211, doi: 10.1016/j.preghy.2012.04.063.

7. Pozo OJ, Marcos J, Khymenets O, et al. SULFATION PATHWAYS: Alternate steroid sulfation pathways targeted by LC-MS/MS analysis of disulfates: application to prenatal diagnosis of steroid synthesis disorders. J Mol Endocrinol. 2018; 61(2): M1-M12, doi: 10.1530/JME-17-0286, indexed in Pubmed: 29459491.

8. Caughey $A B$, Hopkins LM, Norton ME. Chorionic villus sampling compared with amniocentesis and the difference in the rate of pregnancy loss. Obstet Gynecol. 2006; 108(3 Pt 1): 612-616, doi: 10.1097/01. AOG.0000232512.46869.fc, indexed in Pubmed: 16946222.

9. Guo LW, Shackleton $\mathrm{CH}$, Wilson WK. Synthesis of ring B unsaturated estriols. Confirming the structure of a diagnostic analyte for Smith-Lemli-Opitz syndrome. Org Lett. 2001; 3(16): 2547-2550, indexed in Pubmed: 11483057.

10. Jezela-Stanek A, Ciara E, Małunowicz E, et al. Smith-Lemli-Opitz syndrome Collaborative Group. Differences between predicted and established diagnoses of Smith-Lemli-Opitz syndrome in the Polish population: underdiagnosis or loss of affected fetuses? J Inherit Metab Dis. 2010; 33 Suppl 3: S241-S248, doi: 10.1007/s10545-010-9132-4, indexed in Pubmed: 20556518.

11. Choden T, Mandaliya R, Charabaty $A$, et al. Monitoring inflammatory bowel disease during pregnancy: Current literature and future challenges. World J Gastrointest Pharmacol Ther. 2018; 9(1): 1-7, doi: 10.4292/wjgpt.v9.i1.1, indexed in Pubmed: 29430322.

12. Stephansson $\mathrm{O}$, Larsson $\mathrm{H}$, Pedersen $\mathrm{L}$, et al. Crohn's disease is a risk factor for preterm birth. Clin Gastroenterol Hepatol. 2010; 8(6): 509-515, doi: 10.1016/j.cgh.2010.02.014, indexed in Pubmed: 20202483.

13. Cappell MS, Colon VJ, Sidhom OA. A study at 10 medical centers of the safety and efficacy of 48 flexible sigmoidoscopies and 8 colonoscopies during pregnancy with follow-up of fetal outcome and with comparison to control groups. Dig Dis Sci. 1996; 41(12): 2353-2361, doi: 10.1007/bf02100127, indexed in Pubmed: 9011442.

14. Siddiqui I, Majid H, Abid S. Update on clinical and research application of fecal biomarkers for gastrointestinal diseases. World J Gastrointest Pharmacol Ther. 2017; 8(1):39-46, doi: 10.4292/wjgpt.v8.i1.39, indexed in Pubmed: 28217373.

15. Julsgaard M. Fecal Calprotectin Is Not Affected by Pregnancy: Clinical Implications for the Management of Pregnant Patients with Inflammatory Bowel Disease. Inflamm Bowel Dis. 2017; 23(7): 1240-1246.

16. Dai $C$, Jiang $M$, Sun MJ. Fecal markers in the management of inflammatory bowel disease. Postgrad Med. 2018; 130(7): 597-606, doi: 10.1080/00325481.2018.1503919, indexed in Pubmed: 30063872.

17. Aass $C$, Norheim I, Eriksen EF, et al. Single unit filter-aided method for fast proteomic analysis of tear fluid. Anal Biochem. 2015; 480: 1-5, doi: 10.1016/j.ab.2015.04.002, indexed in Pubmed: 25862084.

18. Zhou L, Beuerman RW. Tear analysis in ocular surface diseases. Prog Retin Eye Res. 2012; 31(6): 527-550, doi: 10.1016/j.preteyeres.2012.06.002, indexed in Pubmed: 22732126.
19. Kan S, Acar U, Kizilgul M, et al. Tear Film and Ocular Surface Evaluation in Gestational Diabetes Mellitus. Semin Ophthalmol. 2018; 33(3): 402-406, doi: 10.1080/08820538.2016.1250919, indexed in Pubmed: 28005448.

20. Gutierrez HL, Hund L, Shrestha S, et al. Ethylglucuronide in maternal hair as a biomarker of prenatal alcohol exposure. Alcohol. 2015; 49(6): 617623, doi: 10.1016/j.alcohol.2015.06.002, indexed in Pubmed: 26260252.

21. Jones TB, Bailey BA, Sokol RJ. Alcohol use in pregnancy: insights in screening and intervention for the clinician. Clin Obstet Gynecol. 2013; 56(1): 114-123, doi: 10.1097/GRF.0b013e31827957c0, indexed in Pubmed: 23314712.

22. Boscolo-Berto R, Favretto D, Cecchetto G, et al. Sensitivity and specificity of EtG in hair as a marker of chronic excessive drinking: pooled analysis of raw data and meta-analysis of diagnostic accuracy studies. Ther Drug Monit. 2014; 36(5): 560-575, doi: 10.1097/FTD.0000000000000063, indexed in Pubmed: 24577122.

23. Mughal S, Siddiqui W. Depression, Postpartum. StatPearls, Treasure Island (FL) 2018.

24. Stalder T, Kirschbaum C. Analysis of cortisol in hair--state of the art and future directions. Brain Behav Immun. 2012; 26(7): 1019-1029, doi: 10.1016/j.bbi.2012.02.002, indexed in Pubmed: 22366690.

25. Caparros-Gonzalez RA, Romero-Gonzalez B, Strivens-Vilchez H, et al. Hair cortisol levels, psychological stress and psychopathological symptoms as predictors of postpartum depression. PLoS One. 2017; 12(8): e0182817, doi: 10.1371/journal.pone.0182817, indexed in Pubmed: 28846691.

26. Dunkel Schetter C, Tanner L. Anxiety, depression and stress in pregnancy: implications for mothers, children, research, and practice. Curr Opin Psychiatry. 2012; 25(2): 141-148, doi: 10.1097/YCO.0b013e3283503680, indexed in Pubmed: 22262028.

27. Massey AJ, Campbell BK, Raine-Fenning N, et al. Relationship between hair and salivary cortisol and pregnancy in women undergoing IVF. Psychoneuroendocrinology. 2016; 74: 397-405, doi: 10.1016/j.psyneuen.2016.08.027, indexed in Pubmed: 27756033.

28. Klevay LM, Christopherson DM, ShulerTR. Hair as a biopsy material: trace element data on one man over two decades. Eur J Clin Nutr. 2004; 58(10): 1359-1364, doi: 10.1038/sj.ejcn.1601975, indexed in Pubmed: 15069459.

29. Li Z, Huo W, Li Z, et al. Association between titanium and silver concentrations in maternal hair and risk of neural tube defects in offspring: A case-control study in north China. Reprod Toxicol. 2016; 66: 115-121, doi: 10.1016/j.reprotox.2016.10.006, indexed in Pubmed: 27989884.

30. Jin Xi, Tian X, Liu Z, et al. Maternal exposure to arsenic and cadmium and the risk of congenital heart defects in offspring. Reprod Toxicol. 2016; 59: 109-116, doi: 10.1016/j.reprotox.2015.12.007, indexed in Pubmed: 26743994.

31. Liu Z, Lin Y, Tian X, et al. Association between maternal aluminum exposure and the risk of congenital heart defects in offspring. Birth Defects Res A Clin Mol Teratol. 2016; 106(2): 95-103, doi: 10.1002/bdra.23464, indexed in Pubmed: 26707789.

32. Chiandetti A, Hernandez G, Mercadal-Hally M, et al. Prevalence of prenatal exposure to substances of abuse: questionnaire versus biomarkers. Reprod Health. 2017; 14(1): 137, doi: 10.1186/s12978-017-0385-3, indexed in Pubmed: 29070078.

33. Koss CA, Natureeba P, Mwesigwa J, et al. Hair concentrations of antiretrovirals predict viral suppression in HIV-infected pregnant and breastfeeding Ugandan women. AIDS. 2015; 29(7): 825-830, doi: 10.1097/QAD.0000000000000619, indexed in Pubmed: 25985404.

34. Pink R, Simek J, Vondrakova J, et al. SALIVA AS A DIAGNOSTIC MEDIUM Biomedical Papers. 2009; 153(2): 103-110, doi: 10.5507/bp.2009.017.

35. Kaczor-Urbanowicz K, Carreras-Presas CM, Aro K, et al. Saliva diagnostics - Current views and directions. Exp Biol Med. 2016; 242(5):459-472, doi: $10.1177 / 1535370216681550$.

36. Georgiou HM, Di Quinzio MKW, Permezel M, et al. Predicting Preterm Labour: Current Status and Future Prospects. Dis Markers. 2015; 2015: 435014, doi: 10.1155/2015/435014, indexed in Pubmed: 26160993.

37. Priya B, Mustafa MD, Guleria K, et al. Salivary progesterone as a biochemical marker to predict early preterm birth in asymptomatic high-risk women. BJOG. 2013; 120(8): 1003-1011, doi: 10.1111/1471-0528.12217, indexed in Pubmed: 23551599.

38. Castles A, Adams EK, Melvin CL, et al. Effects of smoking during pregnancy. Five meta-analyses. Am J Prev Med. 1999; 16(3): 208-215, indexed in Pubmed: 10198660.

39. Benowitz N, Dempsey D. Pharmacotherapy for smoking cessation during pregnancy. Nicotine Tob Res. 2004; 6 Suppl 2: S189-S202, doi: 10.1080/14622200410001669169, indexed in Pubmed: 15203821. 
40. Windsor R, Woodby L, Miller T, et al. Effectiveness of Agency for Health Care Policy and Research clinical practice guideline and patient education methods for pregnant smokers in Medicaid maternity care. American Journal of Obstetrics and Gynecology. 2000; 182(1): 68-75, doi: 10.1016/s0002-9378(00)70492-3.

41. Dietz PM, Homa D, England LJ, et al. Estimates of nondisclosure of cigarette smoking among pregnant and nonpregnant women of reproductive age in the United States. Am J Epidemiol. 2011; 173(3): 355-359, doi: 10.1093/aje/kwq381, indexed in Pubmed: 21178103.

42. Benowitz NL. Cotinine as a biomarker of environmental tobacco smoke exposure. Epidemiol Rev. 1996; 18(2): 188-204, doi: 10.1093/oxfordjournals.epirev.a017925, indexed in Pubmed: 9021312.

43. Polanska K, Krol A, Kaluzny P, et al. Estimation of Saliva Cotinine Cut-Off Points for Active and Passive Smoking during Pregnancy-Polish Mother and Child Cohort (REPRO_PL). Int J Environ Res Public Health. 2016; 13(12), doi: 10.3390/ijerph13121216, indexed in Pubmed: 27941658.
44. Shang $M$, Zhao J, Yang $L$, et al. Oxidative stress and antioxidant status in women with gestational diabetes mellitus diagnosed by IADPSG criteria. Diabetes Res Clin Pract. 2015; 109(2): 404-410, doi: 10.1016/j. diabres.2015.05.010, indexed in Pubmed: 26025697.

45. Zygula A, Kosinski $P$, Zwierzchowska $A$, et al. Oxidative stress markers in saliva and plasma differ between diet-controlled and insulin-controlled gestational diabetes mellitus. Diabetes Res Clin Pract. 2019; 148: 72-80, doi: 10.1016/j.diabres.2018.11.021, indexed in Pubmed: 30529575.

46. Łopucki M, Wawrzykowski J, Gęca T, et al. Preliminary analysis of the protein profile in saliva during physiological term and preterm delivery. Mol Med Rep. 2018; 17(6): 8253-8259, doi: 10.3892/mmr.2018.8909, indexed in Pubmed: 29693144.

47. DuY, Zhang W, Wang ML. An On-Chip Disposable Salivary Glucose Sensor for Diabetes Control. J Diabetes Sci Technol. 2016; 10(6): 1344-1352, doi: 10.1177/1932296816642251, indexed in Pubmed: 27053256. 